Louis Miller (National Institute of Allergy and Infectious Diseases) and Stephen Hoffman (Naval Medical Research Institute) review progress toward developing malaria vaccines. They argue that multiple antigens from different stages may be needed to protect the diverse populations at risk, and that an optimal vaccine would induce immunity against all stages. Vaccines for African children, in whom the major mortality occurs, must induce immunity against asexual blood stages.

\title{
Research toward vaccines against malaria
}

Malaria is one of the major causes of disease and death between the Tropic of Cancer and Tropic of Capricorn. Plasmodium falciparum has an especially profound impact on infants and children in sub-Saharan Africa, where its effect on health is increasing as chloroquine resistance spreads across the continent. We believe that vaccination against $P$. falciparum is the intervention with the greatest potential to reduce malaria-associated severe morbidity and mortality in areas with the most intense transmission and that it may do so without necessarily preventing blood stage infection. Malaria vaccines also would be the optimal method for preventing malaria in travelers to countries where malaria is transmitted. Such vaccines will have to entirely prevent the development of any clinical manifestations of infection with $P$. falciparum and of the second most common human malaria parasite, $P$. vivax. This will require preventing blood stage infection.

Infants and young children at risk from $P$. falciparum in Africa and nonimmune travelers to areas endemic for $P$. falciparum and $P$. vivax represent the extremes of target groups in whom malaria vaccines would be useful. Even in countries where the overall numbers of afflicted individuals are not as great as in Africa, malaria wreaks its havoc in many epidemiologic settings and population groups, dramatically undermines the productivity of workers, and drains national budgets. From the Amazon basin to the Indian subcontinent, Southeast Asia, and Oceania, malaria is often a major national public health problem. In these settings, vaccines offer the greatest potential for reducing malaria's debilitating effects. The spread of drug-resistant $P$. falciparum and the recent emergence and spread of chloroquine-resistant $P$. vivax have created an increasing urgency for effective malaria vaccines.

\section{Life cycle and targets of immunity}

Understanding the life cycle (Fig. 1) of the parasite is fundamental to all efforts to develop vaccines-efforts that in the end focus on reducing the effects of the asexual blood stage,

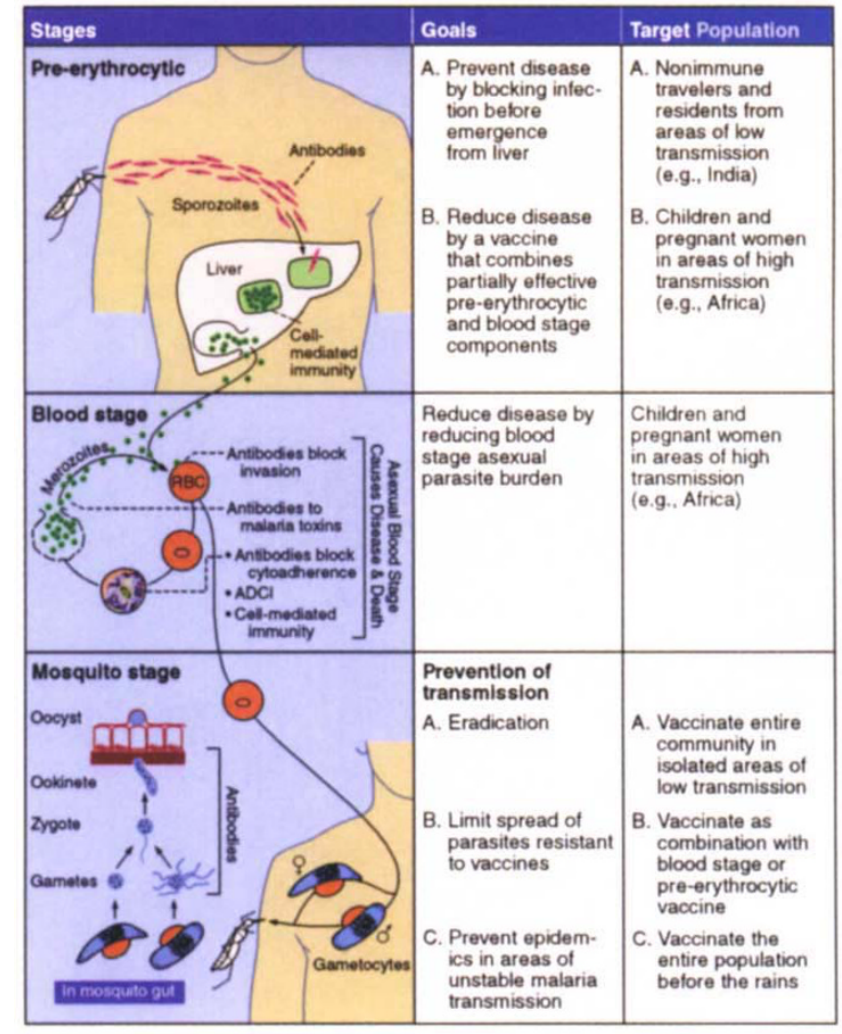

Fig. 1 Multiple vaccines may need to be developed, each for a different population. Each may contain multiple antigens from one stage or combine antigens from different stages. Different formulations and presentations may be needed for antibody or cellular immunity. the only stage of the life cycle that causes disease. The stages before the asexual blood stage are lumped together and called pre-erythrocytic. A small proportion of the asexual blood stages differentiate into sexual stages, the gametocytes in red cells that infect mosquitoes; vaccines to the mosquito stages are called transmission-blocking vaccines. The parasites' multistage life cycle and the fact that immune responses that recognize one stage often do not affect the next stage have made vaccine development for malaria more difficult than for antiviral and antibacterial vaccines.

The pre-erythrocytic stages include sporozoites and parasites that develop in hepatic parenchymal cells. Sporozoites, after inoculation by the mosquito, spend only a few minutes in the blood stream before they invade hepatocytes, in which development takes a minimum of five and a half days in $P$. falciparum. Antibodies to sporozoites block their infection of hepatocytes. The liver stage in rodent malarias, and probably in human malarias, is attacked primarily by $\mathrm{CD}^{+} \mathrm{T}$ cells, but also by $\mathrm{CD}^{+} \mathrm{T}$ cells. These cells can directly eliminate the infected cells or secrete interferon (IFN)- $\gamma$ that induces nitric oxide-dependent killing within hepatocytes. Killing all parasites during the sporozoite stage or during the hepatic infection prevents all disease. Thus, stand-alone vaccines against pre-erythrocytic stages are being developed for protection of travelers. They may also have a role in the general population in countries with low endemicity such as India. Their application on their own in areas with intense transmission is considered by some to be inadvisable. There is a concern that if the vaccines were too good, they would prevent the development of naturally acquired anti-blood stage immunity, and if the anti-pre erythrocytic immunity waned with time, the immunized population would be left without any immunity and could suffer an increased incidence of severe disease and death. This problem would be mitigated if a pre-erythrocytic 


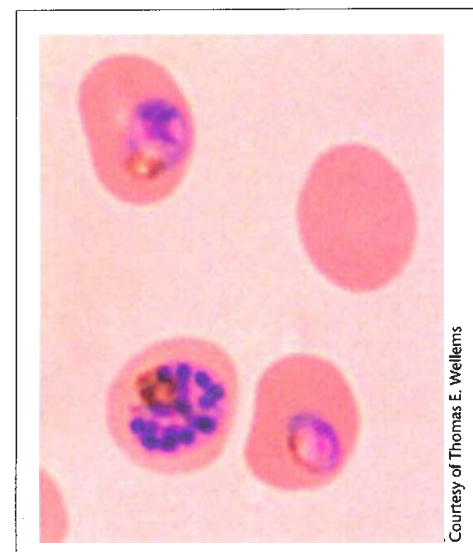

Human red cells infected with Plasmodium falciparum. stage vaccine were combined with a blood stage vaccine, a strategy some consider to be optimal.

The blood stage begins when parasites leave liver cells and invade red cells. The parasite develops and multiplies within the red cell; 16 or more merozoites appear 48 hours after invasion (in $P$. falciparum and $P$. vivax). The infected red cell lyses, releasing merozoites, each capable of invading other red cells. The parasitemia can increase ten-fold

each 48 hours. No disease occurs until the parasitemia reaches a level critical for induction of fever and shaking chills. The serious complications of anemia and coma appear later in the infection as the parasitemia rises. The current vaccine approach is to design blood stage vaccines that will prime the individual for boosting of immunity by the parasite during each infection, thereby controlling parasitemia before serious complications occur. There are data indicating that disease in young children in Africa is influenced by the intensity of transmission (number of infectious bites per year), the child's genetics, and perhaps virulence factors in $P$. falciparum ${ }^{1}$. For example, in many areas, cerebral malaria (coma and seizures with high mortality) is uncommon before two years of age $\mathrm{e}^{2}$. In low-transmission areas ( $<15$ bites/individual by infected mosquitoes per year), severe anemia occurs early in life and cerebral malaria later, suggesting a developmental susceptibility to cerebral malaria in children ${ }^{2}$. In high-transmission areas $(>100$ bites/individual by infected mosquitoes per year), severe anemia occurs early in life and cerebral malaria occurs infrequently ${ }^{1,3}$, possibly because children become immune before the age at which cerebral malaria occurs. Despite these observations, the pathogenesis of disease in African children is poorly defined. Current development efforts assume that reducing parasitemia will limit all complications of malaria. This assumption may be wrong, and some complications may be worsened by vaccination. The cost of vaccine trials, however, demands this simplifying assumption to test the multiple antigens with different formulations that will be needed for blood stage vaccine development. The young children used to test selected candidates need to be followed closely for increased complications after vaccination.

Throughout the blood stage of the infection, a percentage of asexual blood stage parasites convert to gametocytes that are infectious to mosquitoes. Factors in the mosquito midgut induce gametocytes to transform into gametes and zygotes that develop into ookinetes, the stage that penetrates the mosquito's chitinous peritrophic membrane and

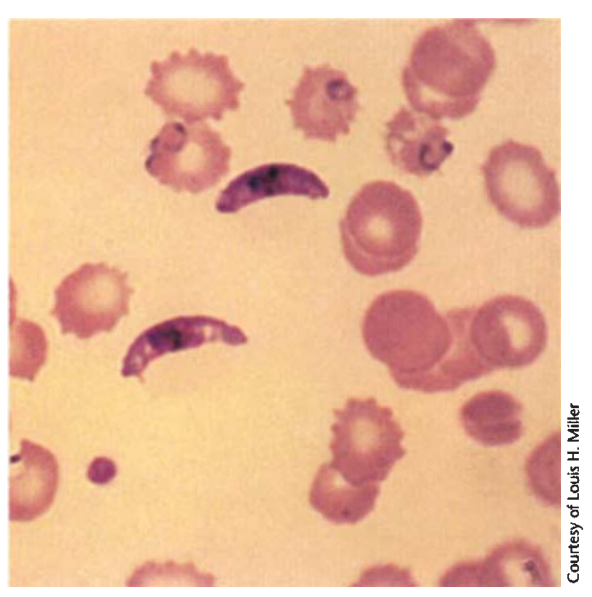

Human red cells infected with gametocytes (sexual stage) and ring forms (asexual stage) of Plasmodium falciparum. invades midgut epithelial cells. Parasites on the midgut epithelium develop into thousands of sporozoites that invade the salivary glands to transmit the infection while feeding on people. Transmission-blocking vaccines under development contain gamete, zygote and ookinete surface proteins and secreted proteins from ookinetes. They induce antibodies that, when ingested with the blood meal, block fertilization, development, penetration of the peritrophic membrane or invasion of midgut epithelium. Such vaccines would block transmission of mutant parasites resistant to drugs or to vaccines against other stages. Furthermore, they could be used for epidemic control in areas of unstable transmission. The third use, in combination with other interventions such as insecticides, would be for malaria eradication in island communities (such as Sri Lanka) where transmission is low. Several constructs that contain a surface protein on ookinetes, Pfs 25 , are under development and are being tested in normal volunteers.

Unlike most viral vaccines, malaria vaccines will be produced as subunits that will contain only a few of the estimated 5,000-7,000 proteins in the parasite or parts of these proteins. Below, we identify obstacles to successful subunit vaccine development for pre-erythrocytic and blood stage vaccines and present our perspective on how to overcome them. For those interested in the details of antigens and strategies for vaccine development, see refs. $4 \& 5$.

\section{Pre-erythrocytic stage vaccines}

Immunization of mice and humans with radiation-attenuated sporozoites of rodent and human malaria parasites, respectively, induces complete protection against the pathologic and clinical manifestations of the infection. The protective immunity prevents the parasites from emerging from the liver into the bloodstream, is not strain specific, and lasts in humans for at least nine months (ref. 6 for review). This would be an ideal vaccine for travelers but is impractical because it requires exposure to thousands of infected, irradiated mosquitoes.

Efforts for 30 years have focused on characterizing the protective immune responses and their antigenic targets and developing vaccine delivery systems to induce comparable immunity. Antibodies induced by irradiated sporozoites have modest antisporozoite inhibitory activity in mice and humans, but protection in mice is always dependent on $\mathrm{CD}^{+} \mathrm{T}$ lymphocytes. These $\mathrm{T}$ cells eliminate parasite-infected hepatocytes from culture. The $\mathrm{CD} 8^{+} \mathrm{T}$ cell immunity may be the result of lysis of infected hepatoctyes by cytotoxic T lymphocytes (CTL), but the T cells also release IFN- $\gamma$, which induces the infected hepatocyte to produce nitric oxide that eliminates the infected cells. Depending on the individual and the antigen, both mechanisms of killing are probably important ${ }^{6}$. Humans immunized with irradiated $P$. falciparum sporozoites have been shown to have $\mathrm{CD} 8^{+}$CTLs against four different $P$. falciparum proteins expressed in infected hepatoctyes (refs. $6 \& 7$ for review); subunit vac- 
cines of the rodent malaria parasite orthologues of three of these induce $\mathrm{CD}^{+} \mathrm{T}$ cell-dependent ${ }^{8-10}$ and, in one case, also IFN- $\gamma$ - and nitric oxide-dependent protection in mice ${ }^{10}$. It must be remembered, however, that irradiated sporozoites may induce immunity against hundreds or thousands of proteins expressed in infected hepatocytes, not just the handful currently under study.

The challenge, as in vaccinology in general, has been to develop subunit vaccines that induce protective $\mathrm{CD}^{+} \mathrm{T}$ cell responses in humans. Intramuscular immunization with recombinant, attenuated vaccinia expressing several liver stage $P$. falciparum proteins induced modest CTLs but minimal protection ${ }^{11}$. Based on work in mice and rhesus monkeys, there is now great hope that ongoing clinical trials with DNA vaccines will demonstrate $\mathrm{CD} 8^{+} \mathrm{T}$ cell-dependent protection in humans ${ }^{12}$. Regardless of the results of these early trials with DNA alone, preliminary studies in mice indicate that DNA vaccine-induced immunity and protection against malaria can be improved by co-administration of plasmids expressing cytokines ${ }^{13}$ and by prime boost approaches ${ }^{14,15}$. It is likely that these and other ${ }^{16}$ approaches will be studied in humans in the next few years. Once the problem of optimal induction of these responses is achieved, a successful vaccine will have to include adequate $\mathrm{CD}^{+} \mathrm{T}$ cell epitopes to overcome the problems of genetic restriction of $\mathrm{CD}^{+} \mathrm{T}$ cell responses $^{7}$, variation of epitopes in the field ${ }^{7}$, and perhaps even epitope antagonism ${ }^{17}$.

Although the protective immunity induced by irradiated sporozoites is probably primarily mediated by $\mathrm{T}$ cells, the best $(80-100 \%)$, most consistent and least genetically restricted protection achieved in rodents has been with vaccines that induce antibodies that prevent sporozoite invasion of hepatocytes ${ }^{18}$. Monoclonal antibodies against a tandem repeat domain on the circumsporozoite protein (CSP) inhibit sporozoite invasion of hepatocytes in vitro and passively transfer protection to mice and monkeys. This domain is conserved among all $P$. falciparum isolates. Based on these studies, clinical trials with PfCSP repeat region recombinant protein and synthetic peptide vaccines have been conducted for more than a decade. The principle that such vaccines could protect was proved in the first trials in which aluminum hydroxide was used as adjuvant; two of the four volunteers with the highest levels of antibodies were completely protected ${ }^{19,20}$. Increasing the complexity of the immunogen and including more potent adjuvants has resulted in a steady increase in immunogenicity. Recently, RTS,S-a hybrid containing the central repeats and most of the $\mathrm{C}$-terminus of the PfCSP fused to hepatitis B surface antigen in a complex adjuvant mixture (SBAS4) ${ }^{31}$-completely protected six of seven volunteers against sporozoite challenge three weeks after the last immunization. Volunteers immunized with RTS,S in other adjuvants had little protection. Anti-PfCSP antibodies were extremely high in most protected volunteers but some unprotected volunteers had comparable antibody titers. The six protected volunteers were challenged approximately six months after the first challenge and one was protected $^{22}$. A field study of RTS,S-SBAS4 in The Gambia and additional clinical trials of RTS,S and of a PfCSP multiple antigenic peptide vaccine $\mathrm{e}^{18}$ are now in progress.

A ligand that binds to a receptor on hepatoctyes has been identified on the CSP and on a second sporozoite surface protein called sporozoite surface protein 2 (SSP2) or throm-

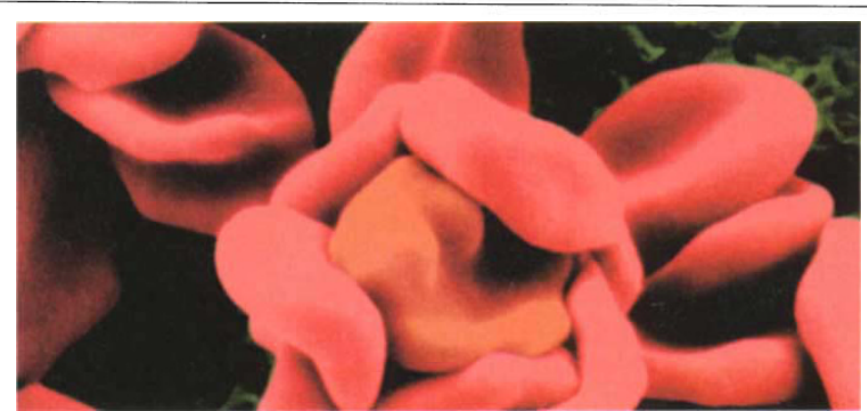

Plasmodium falciparum-infected red cell (center) binding uninfected red cells. Courtesy of Kjell Hultenby \& Carl Johan Treutiger.

bospondin-related anonymous protein (TRAP) (ref. 18). Thus far, efforts to induce highly inhibitory antibodies against this domain, which bears some homology to human thrombospondin, have been unsuccessful. Additional sporozoite surface proteins have been identified, and some have been shown to induce inhibitory antibodies, but to date none has been included in an experimental human vaccine.

During the past decade, the major efforts on pre-erythrocytic malaria vaccine development have focused on inducing antibodies to the sporozoite surface and $C D 8^{+} \mathrm{T}$ cells against infected hepatoctyes; however, there is now considerable interest in protective $\mathrm{CD}^{+} \mathrm{T}$ cell responses against infected hepatocytes. New data indicate that in several strains of mice, protection induced by the irradiated sporozoite vaccine is eliminated by treatment of the mice with anti-CD4 antibodies or anti-CD8 antibodies. $\mathrm{CD}^{+} \mathrm{T}$ cell clones against the PyCSP eliminate infected hepatocytes from culture and adoptively transfer protection ${ }^{23}$. Immunization with linear synthetic peptides from two liver stage proteins induces consistent, genetically restricted, $\mathrm{CD}^{+} \mathrm{T}$ cell- and IFN- $\gamma$-dependent protection in mice ${ }^{24}$. The RTS,S PfCSP vaccine described above is a potent activator of IFN- $\gamma$-producing $\mathrm{CD}^{+} \mathrm{T}$ cells. In addition to antibodies, $\mathrm{CD}^{+} \mathrm{T}$ cells may have played an important role in the protection of volunteers induced by this vaccine.

The irradiated sporozoite vaccine has served as an important model for developing pre-erythrocytic stage vaccines; however, particularly in regard to protective antibodies and $\mathrm{CD}^{+} \mathrm{T}$ cell responses, it seems that we can do better than the model. The challenge for the future is to develop a pre-erythrocytic vaccine capable of inducing not only protective antibodies against sporozoites but also $\mathrm{CD}^{+}$and $\mathrm{CD}^{+} \mathrm{T}$ cell responses against infected hepatocytes, incorporating into these vaccines the important parasite proteins expressed on the sporozoite surface or early in the liver stage identified by the malaria genome project.

\section{Blood stage vaccines}

Two observations make us confident that a blood stage vaccine is possible. First, people in endemic areas become immune after repeated infections. Immune older children and adults have blood stage infections but are usually asymptomatic. Thus, immunity is measured by the absence of disease, not by resistance to reinfection, and the goal of a blood stage vaccine is to prevent disease, not infection. Second, immunization of animals induces protection that is more effective than immunity induced by chronic, repeated infections. For example, monkeys immunized against $P$. knowles $i$ 
parasites in Freund's complete adjuvant can rapidly clear challenge infections with both homologous and heterologous strains ${ }^{25}$

Effective approaches to antigen identification have been through monoclonal antibodies to merozoite surface molecules and organellar proteins and through the use of Triton $\mathrm{X}-114$ to differentially solubilize surface proteins ${ }^{26}$. The most promising candidate to date has been a merozoite surface protein (MSP-1) that was identified by monoclonal antibodies. Vaccination with immunoaffinity-purified MSP-1 from a rodent parasite ${ }^{27}$ and from $P$. falciparum induced protection in mice and monkeys, respectively. Protective monoclonal antibodies reacted with an epidermal growth factor-like domain in the C-terminus [MSP-1(19)]; immunization with recombinant proteins from this region protected against lethal $P$. yoelii in mice and against $P$. falciparum in Aotus monkeys 5 . In MSP-1(19)-immunized mice, immunity is primarily antibody mediated; high titer sera in immunized mice correlates with protection ${ }^{28}$. Other promising antigens and immune mechanisms such as antibody-dependent cellular inhibition $^{29}$ are also under study ${ }^{30}$.

Patarroyo and his team from Colombia identified potential candidate antigens by immunizing Aotus monkeys with peptides linked to bovine serum albumin and challenging them with virulent $P$. falciparum ${ }^{31}$. The three peptides that induced some protection were synthesized as a single 66 amino acid peptide, polymerized and bound to aluminum hydroxide. This vaccine was tested in Latin America, Africa and Asia with evidence of protection in some studies ${ }^{32}$ but not in others ${ }^{33}$. The lack of in vitro correlates of immunity made interpretation of the variable results difficult.

Parasite ligands for invading red cells and for binding of infected red cells to endothelium may be candidates for vaccine development. Apicomplexa usually invade host cells by specific ligands in the apical end. These proteins and the domains within the proteins for red cell binding have been identified in Plasmodium. One domain is conserved in $P$. falciparum and $P$. vivax, although the red cell receptors differ ${ }^{34,35}$. Recombinant proteins from these domains are now being tested for inducing neutralizing antibodies in monkey malarias. One concern is that these receptors may be exposed on the red cell surface for a short period between release of merozoites from one red cell and invasion of another red cell. A further concern is that the receptors are found within an organelle at the apical end and may only be released for binding after the merozoite has attached to a red cell, a step that signals the merozoite to begin the invasion process. A membrane protein on merozoites involved in signaling would be a prime candidate for a vaccine, but none has been identified to date.

Infected red cells bind to endothelium in a receptor-specific manner to protect the infected red cell from the killing effects of the spleen. A number of endothelial receptors have been identified; the parasite ligands for these receptors are encoded by a highly variant protein with up to 150 copies per parasite genome. Curiously, the parasite ligands for binding endothelium have been found on a domain that contains homology to the red cell binding domain involved in invasion $^{36}$. Each variant protein on the red cell surface has multiple copies of this domain. One of these domains binds endothelium via $\mathrm{CD} 36$ (ref. 37), which is thought to be a receptor to which most infected red cells bind. As this domain is less variable than other parts of the molecule and binds to this common receptor on endothelium, it may have a conserved structure. Thus, it may be possible to develop an immunogen of this domain that induces antibody to block endothelial attachment. Although this is counterintuitive (variation is designed to escape immunity), the epitopes around this domain may not normally induce immunity because they are cryptic, that is, not immunogenic during infection, but protection can be induced after vaccination.

If many antigens derived from blood stage parasites have been identified and some have been shown to be effective after vaccination of mice and monkeys, why have they not been tested in humans? The main obstacle to vaccine development has been the absence of industrial interest in blood stage vaccines because they do not expect profits that would warrant the investment. Thus, a gap exists between the research laboratories that lack the costly facilities to produce blood stage vaccines for human trials, and industry that has the capability, but not the motivation, to produce them. Transition from a research laboratory setting to vaccine production for human trials will require a different mind set for the scientist, and costly facilities ${ }^{5}$. The National Institutes of Health is in the process of setting up a unit to attempt to effect this transition.

\section{Looking ahead}

The most exciting period for the scientific community will be when we extensively test vaccines in endemic countries, and particularly in Africa. We will be studying the effect of vaccines in the natural host and under conditions in which the host is repeatedly infected. One concern for an antibody-based vaccine is the selection of parasites resistant to the vaccine. We know that every parasite protein exposed to the immune system has many polymorphisms. It is possible that structural requirements for critical domains limit the flexibility of the parasite, especially if the vaccine contains multiple epitopes. Combining a transmission-blocking vaccine with a blood stage vaccine may prolong the life of a vaccine by reducing transmission.

In addition to antigenic polymorphism, the parasite may have evolved other mechanisms of immune evasion. There may be ways that the parasite drives a nonprotective immune response or induces apoptosis of immune $\mathrm{T}$ cells. The large number of parasite proteins with repeat epitopes suggested to Anders the possibility that crossreactions may interfere with maturation of the immune response to produce high affinity antibodies ${ }^{38}$. The low complexity of many malarial proteins with no fixed globular structure ${ }^{39}$ may also be a mechanism of immune evasion. Hopefully, the globular structure of the vaccine candidates under study such as the C-terminus of MSP-1 and AMA-1 may circumvent these problems.

Another scenario is the possible effect of repeated infections that may give rise to greater immunity in humans after vaccination, more so than we have seen in animal studies. We know that previously infected animals have a secondary response to first immunization. Although vaccination of rhesus monkeys with AMA-1 of $P$. knowlesi afforded minimal protection, the immunized, previously infected animals were strongly protected from reinfection ${ }^{40}$. Thus, infection after immunization was critical to induce high levels of immunity. 


\section{Conclusion}

The malaria community has characterized immune mechanisms responsible for protection, identified numerous target antigens and is on the cutting edge of research in vaccinology. In the coming years, we will use the advances in immunology and vaccinology and discover new potential vaccine targets from the Plasmodium genome project. We know that our goals are achievable because of successful vaccination of humans with irradiated sporozoites and monkeys with blood stage parasites and because of the fact that people become immune. It is now time to make the commitment to GMP vaccine manufacture and human trials, including phase III mortality studies. The seriousness of the human problem demands that we develop this most cost-effective tool.

1. Miller, L.H., Good, M.F. \& Milon, G. Malaria pathogenesis. Science 264, 1878-1883 (1994).

2. Marsh, K. Malaria-a neglected disease? Parasitology 104, 553-569 (1992).

3. Snow, R.W. et al. Relation between severe malaria morbidity in children and level of Plasmodium falciparum transmission in Africa. Lancet 349, 1650-1654 (1997).

4. Hoffman, S.L (ed.) Malaria Vaccine Development: A Multi-Immune Response Approach. Washington, D.C.: ASM Press (1996).

5. Good, M.F., Kaslow, D.C. \& Miller, L.H. Pathways and strategies for developing a malaria blood stage vaccine. Ann. Rev. Immunol. 16, 57-87 (1998).

6. Hoffman, S.L., Franke, E.D., Hollingdale, M.R. \& Druilhe, P. Attacking the infected hepatocyte. In: Malaria Vaccine Development: A Multi-Immune Response Approach, Hoffman, S.L. (ed.) Washington, D.C.: American Society of Microbiology, pp. 35-75 (1996).

7. Doolan, D.L. et al. Degenerate cytotoxic T cell epitopes from Plasmodium falciparum restricted by multiple HLA-A and HLA-B supertype alleles. Immunity 7 97-112 (1997).

8. Aggarwal, A. et al. Oral Salmonella: malaria circumsporozoite recombinants induce specific CD8 ${ }^{+}$cytotoxic T cells. J. Exp. Med. 172, 1083-1090 (1990).

9. Khusmith, S., et al. Protection against malaria by vaccination with sporozoite surface protein 2 plus CS protein. Science 252, 715-718 (1991).

10. Doolan, D.L. et al. Circumventing genetic restriction of protection against malaria with multigene DNA immunization: $\mathrm{CD}^{+}$cell-, interferon--, and nitric oxide-dependent immunity. J. Exp. Med. 183, 1739-1746 (1996).

11. Ockenhouse, C.F. et ol. Phase I/lla safety, immunogenicity, and efficacy of NYVAC-Pf7, a pox-vectored, multiantigen, multistage vaccine candidate for Plasmodium falciparum malaria. J. Infect. Dis. (in the press).

12. Hoffman, S.L. et al. Toward clinical trials of DNA vaccines against malaria. Immunol. Cell Biol. 75, 376-381 (1997).

13. Weiss, W. et al. A plasmid encoding murine GMCSF increases protection conferred by a malaria DNA vaccine. J. Immunol. (1998) (in press).

14. Schneider, J. et al. Enhanced immunogenicity for CD8+ T cell induction and complete protective efficacy of malaria DNA vaccination by boosting with modified vaccinia virus Ankara. Nature Med. 4, 397-402 (1998).

15 Sedegah, M. et al. Boosting with recombinant vaccinia increases immunogenicity and protective efficacy of malaria DNA vaccine. Proc. Natl. Acad. Sci. USA (1998) (in press).

16. Rodrigues, E.G., Zavala, F., Eichinger, D., Wilson, J.M. \& Tsuji, M. Single immunizing dose of recombinant adenovirus efficiently induces CD8+ T cell-mediated protective immunity against malaria. J. Immunol. 158, 1268-1274 (1997).

17. Gilbert, S.C. et al. Association of malaria parasite population structure, HLA, and immunological antagonism. Science 279, 1173-1177 (1998).

18. Sinnis, P. \& Nussenzweig, V. Preventing sporozoite invasion of hepatocytes. In: Malaria Vaccine Development: a Multi-Immune Response Approach, Hoffman, S.L. (ed.) Washington, D.C.: American Society of Microbiology, pp. 15-33 (1996).

19. Herrington, D.A. et al. Safety and immunogenicity in man of a synthetic peptide malaria vaccine against Plasmodium falciparum sporozoites. Nature 328 , 257-259 (1987).

20. Ballou, W.R. et al. Safety and efficacy of a recombinant DNA Plasmodium falciparum sporozoite vaccine. Lancet 1, 1277-1281 (1987).

21 Stoute, J.A. et al. A preliminary evaluation of a recombinant circumsporozoite protein vaccine against Plasmodium falciparum malaria. RTS, S Malaria Vaccine Evaluation Group. N. Engl. J. Med. 336, 86-91 (1997).

22. Stoute, J.A. et al. Long-term efficacy and immune responses following immunization with the RTS, S malaria vaccine. J. Infect. Dis. (1998) (in press).

23. Renia, L. et al. In vitro activity of CD4+ and CD8+ lymphocytes frommice immunized with a synthetic malaria peptide. Proc. Natl. Acad. Sci. USA $\mathbf{8 8}$ 7963-7967 (1991).

24. Wang, R. et al. Protection against malaria by Plasmodium yoelii sporozoite surface protein 1 linear peptide induction of CD4+ T cell- and IFN- $\gamma$-dependent elimination of infected hepatocytes. J. Immunol. 157, 4061-4067 (1996).

25. Mitchell, G.H., Butcher, G.A. \& Cohen, S. Merozoite vaccination against Plasmodium knowlesi malaria. Immunology 29, 397-407 (1975).

26. Smythe, J.A. et al. Identification of two integral membrane proteins of Plasmodium falciparum. Proc. Natl. Acad. Sci. USA 85, 5195-5199 (1988).

27. Holder, A.A. \& Freeman, R.R. Immunization against blood stage rodent malaria using purified parasite antigens. Nature 294, 361-366 (1982).

28. Hirunpetcharat, $C$., et al. Complete protective immunity induced in mice by immunization with the 19-kilodalton carboxyl-terminal fragment of the merozoite surface protein (MSP1 ${ }_{19}$ ) of Plasmodium yoelii expressed in Saccharomyces cerevisiae: correlation of protection with antigen-specific antibody titer, but not with effector CD4+ T cells. I. Immunol. 159, 3400-3411 (1997).

29. Bouharoun-Taylor, H., Oeuvray, C., Lunel, F. \& Druilhe, P. Mechanisms underlying the monocyte-mediated antibody-dependent killing of Plasmodium falciparum asexual blood stages. J. Exp. Med. 182, 409-418 (1995).

30. Holder, A.A. Preventing merozoite invasion of erythrocytes. In: Malaria Vaccine Development: A Multi-Immune Response Approach, Hoffman, S.L. (ed.) Washington, D.C.: American Society of Microbiology, pp. 77-104 (1996).

31. Patarroyo, M.E. et al. Induction of protective immunity against experimental infection with malaria using synthetic peptides. Nature 328, 629-632 (1987)

32. Alonso, P.L. et al. Randomised trial of efficacy of SPf6 vaccine against Plasmodium falciparum malaria in children in southern Tanzania. Lancet 344 , 1175-1181 (1994).

33. d'Allessandro, U. et al. Efficacy trial of malaria vaccine SPf66 in Gambian infants. Lancet 346, 462-467 (1995).

34. Chitnis, C.E. \& Miller, L.H. Identification of the erythrocyte binding domains of Plasmodium vivax and Plasmodium knowlesi proteins involved in erythrocyte in vasion. J. Exp. Med. 180, 497-506 (1994).

35. Sim, B.K.L., Chitnis, C.E., Wasniowska, K., Hadley, T.J. \& Miller, L.H. Receptor and ligand domains for invasion of erythrocytes by Plasmodium falciparum. Science 264, 1941-1944 (1994).

36. Su, X.-Z. et al. The large diverse gene family var encodes proteins involved in the cytoadherence and antigenic variation of Plasmodium falciparum-infected erythrocytes. Cell 82, 89-100 (1995).

37. Baruch, D.I. et al. Identification of a region of PfEMP1 that mediates adherence of Plasmodium falciparum infected erythrocytes to CD36: conserved function with variant sequence. Blood 90, 3766-3775 (1997).

38. Anders, R.F. Multiple cross-reactivities amongst antigens of Plasmodium falciparum impair the development of protective immunity against malaria. Parasite Immunol. 8, 529-539 (1986).

39. Wooton, J.C. Non-globular domains in protein sequence: automated segmentation using complexity measures. Computers Chem. 18, 269-285 (1994)

40. Deans, J.A., et al. Vaccination trials in rhesus monkeys with a minor, invariant, Plasmodium knowlesi 66 kD merozoite antigen. Parasite Immunol. 10, 535-552 (1988).

${ }^{1}$ Laboratory of Parasitic Diseases, National Institute of Allergy and

Infectious Diseases, National Institutes of Health, Bethesda,

Maryland 20892 USA

${ }^{2}$ Malaria Program, Naval Medical Research Institute, Bethesda,

Maryland 20889-5607 USA

Correspondence should be addressed to L.H.M.

e-mail: louis_miller@nih.gov

The opinions and assertions herein are those of the author (S.L.H.) and are not to be construed as official or as reflecting the views of the U.S. Navy or naval services at large. This work was supported in part by STO F 6.3a63002AA0101HFX.1433. 